SIT-LP-05/07

hep-th/0507288

July, 2005

\title{
On supersymmetry algebra based on a spinor-vector generator
}

\author{
Kazunari Shima * and Motomu Tsuda ${ }^{\dagger}$ \\ Laboratory of Physics, Saitama Institute of Technology \\ Okabe-machi, Saitama 369-0293, Japan
}

\begin{abstract}
We study the unitary representation of supersymmetry (SUSY) algebra based on a spinor-vector generator for both massless and massive cases. A systematic linearization of nonlinear realization for the SUSY algebra is also discussed in the superspace formalism with a spinor-vector Grassmann coordinate.
\end{abstract}

\footnotetext{
*e-mail: shima@sit.ac.jp

†e-mail: tsuda@sit.ac.jp
} 
Both linear (L) [1] and nonlinear (NL) [2] supersymmetry (SUSY) are realized based on a SUSY algebra where spinor generators are introduced in addition to Poincaré generators. The relation between the L and the NL SUSY, i.e., the algebraic equivalence between various (renormalizable) spontaneously broken L supermultiplets and a NL SUSY action [2] in terms of a Nambu-Goldstone (NG) fermion has been investigated by many authors [3]-[6].

An extension of the Volkov-Akulov (VA) model [2] of NL SUSY based on a spinor-vector generator, called the spin-3/2 SUSY, hitherto, and its NL realization in terms of a spin-3/2 NG fermion have been constructed by N.S. Baaklini [7]. From the spin-3/2 NL SUSY model, L realizations of the spin-3/2 SUSY are suggested as corresponding supermultiplets to a spin-3/2 NL SUSY action [7] through a linearization, although those have not yet known at all. Also the linearization of the spin-3/2 NL SUSY is useful from the viewpoint towards constructing a SUSY composite unified theory based on $\mathrm{SO}(10)$ super-Poincaré (SP) group (the superon-graviton model $(\mathrm{SGM})$ ) [8]. Indeed, it may give new insight into an analogous mechanism with the super-Higgs one [9] for high spin fields which appear in SGM (up to spin-3 fields).

In this letter, we investigate the unitary representation of the spin-3/2 SUSY algebra in [7] towards the linearization of the spin-3/2 NL SUSY. The role of the spinor-vector generator as creation and annihilation operators for helicity states is discussed explicitly. For both massless and massive representaions for the spin-3/2 SUSY algebra, we show examples of L supermutiplet-structure induced from those representations. We also discuss on a systematic linearization of the spin-3/2 NL SUSY in the superspace formalism with a spinor-vector Grassmann coordinate.

Let us begin with the brief review of the spin-3/2 SUSY algebra and its NL realization in terms of the spin-3/2 NG fermion. The spin-3/2 SUSY algebra in [7], which satisfies all the Jacobi identities, is introduced based on a (Majorana) spinor-vector generator $Q_{\alpha}^{a}$ as $\ddagger$

$$
\begin{aligned}
& \left\{Q_{\alpha}^{a}, Q_{\beta}^{b}\right\}=i \epsilon^{a b c d}\left(\gamma_{5} \gamma_{c} C\right)_{\alpha \beta} P_{d}, \\
& {\left[Q_{\alpha}^{a}, P^{b}\right]=0} \\
& {\left[Q_{\alpha}^{a}, J^{b c}\right]=\frac{1}{2}\left(\sigma^{b c}\right)_{\alpha}^{\beta} Q_{\beta}^{a}+i\left(\eta^{a b} Q_{\alpha}^{c}-\eta^{a c} Q_{\alpha}^{b}\right),}
\end{aligned}
$$

where $P^{a}$ and $J^{a b}$ are taranslational and Lorentz generators of the Poincaré group.

\footnotetext{
${ }^{\ddagger}$ Minkowski spacetime indices are denoted by $a, b, \cdots=0,1,2,3$ and four-component spinor indices are $\alpha, \beta, \cdots=(1),(2),(3),(4)$. The Minkowski spacetime metric is $\frac{1}{2}\left\{\gamma^{a}, \gamma^{b}\right\}=\eta^{a b}=$ $(+,-,-,-)$ and $\sigma^{a b}=\frac{i}{2}\left[\gamma^{a}, \gamma^{b}\right]$. We also use the spinor representation of the $\gamma$ matrices, $\gamma^{0}=$ $\left(\begin{array}{ll}0 & I \\ I & 0\end{array}\right), \gamma^{i}=\left(\begin{array}{cc}0 & -\sigma^{i} \\ \sigma^{i} & 0\end{array}\right)$ with $\sigma^{i}$ being the Pauli matrices, $\gamma_{5}=i \gamma^{0} \gamma^{1} \gamma^{2} \gamma^{3}$ and the charge conjugation matrix, $C=-i \gamma^{0} \gamma^{2}$.
} 
The NL realization of the spin-3/2 SUSY which reflects Eq.(1) is given by introducing the spin-3/2 (Majorana) NG fermion $\psi^{a}[7]$. Indeed, supertranslations of the $\psi^{a}$ and the Minkowski coordinate $x^{a}$ parametrized by a global (Majorana) spinor-vector parameter $\zeta^{a}$ are defined as

$$
\begin{aligned}
\delta \psi^{a} & =\frac{1}{\kappa} \zeta^{a}, \\
\delta x^{a} & =\kappa \epsilon^{a b c d} \bar{\psi}_{b} \gamma_{5} \gamma_{c} \zeta_{d},
\end{aligned}
$$

where $\kappa$ is a constant whose dimension is (mass) $)^{-2}$. Eq.(4) means a NL SUSY transformation of $\psi^{a}$ at a fixed spacetime point,

$$
\delta_{Q} \psi^{a}=\frac{1}{\kappa} \zeta^{a}-\kappa \epsilon^{b c d e} \bar{\psi}_{b} \gamma_{5} \gamma_{c} \zeta_{d} \partial_{e} \psi^{a}
$$

which gives the closed off-shell commutator algebra,

$$
\left[\delta_{Q 1}, \delta_{Q 2}\right]=\delta_{P}\left(\Xi^{a}\right)
$$

where $\delta_{P}\left(\Xi^{a}\right)$ means a translation with a generator $\Xi^{a}=2 \epsilon^{a b c d} \bar{\zeta}_{1 b} \gamma_{5} \gamma_{c} \zeta_{2 d}$.

As parallel discussions in the VA model of the ordinary (spin-1/2) NL SUSY [2], a spin-3/2 NL SUSY invariant differential one-form is given by

$$
\begin{aligned}
\omega^{a} & =d x^{a}+\kappa^{2} \epsilon^{a b c d} \bar{\psi}_{b} \gamma_{5} \gamma_{c} d \psi_{d} \\
& =\left(\delta_{b}^{a}+\kappa^{2} \epsilon^{a c d e} \bar{\psi}_{c} \gamma_{5} \gamma_{d} \partial_{b} \psi_{e}\right) d x^{b} \\
& =\left(\delta_{b}^{a}+t^{a}{ }_{b}\right) d x^{b} \\
& =w^{a}{ }_{b} d x^{b}
\end{aligned}
$$

and also a NL SUSY invariant action constructed from Eq.(7) has the form

$$
\begin{aligned}
S= & -\frac{1}{2 \kappa^{2}} \int \omega_{0} \wedge \omega_{1} \wedge \omega_{2} \wedge \omega_{3} \\
= & -\frac{1}{2 \kappa^{2}} \int d^{4} x|w| \\
= & -\frac{1}{2 \kappa^{2}} \int d^{4} x\left[1+t^{a}{ }_{a}+\frac{1}{2}\left(t^{a}{ }_{a} t^{b}{ }_{b}-t^{a}{ }_{b} t^{b}{ }_{a}\right)\right. \\
& \left.-\frac{1}{3 !} \epsilon_{a b c d} \epsilon^{e f g d} t^{a}{ }_{e} t^{b}{ }_{f} t^{c}{ }_{g}-\frac{1}{4 !} \epsilon_{a b c d} \epsilon^{e f g h} t^{a}{ }_{e} t^{b}{ }_{f} t^{c}{ }_{g} t^{d}{ }_{h}\right] .
\end{aligned}
$$

The second term in Eq.(8), i.e., $-\left(1 / 2 \kappa^{2}\right) t^{a}{ }_{a}$, is the ordinary Rarita-Schwinger kinetic term for $\psi^{a}$. 
Since (spontaneously broken) spin-3/2 L supermultiplets are suggested from a linearization of the spin-3/2 NL SUSY, we investigate the structure of L supermultiplets induced from the spin-3/2 SUSY algebra (1) to (3). For this purpose, we first focus on the relation (3) and discuss on the role of the spinor-vector generator $Q_{\alpha}^{a}$ as creation and annihilation operators for helicity states. When we choose the moving direction of a massless particle as the 3-axis, Eq.(3) for the helicity operator $J^{12}$ becomes

$$
\begin{aligned}
& {\left[Q_{\alpha}^{0}, J^{12}\right]= \pm \frac{1}{2} Q_{\alpha}^{0},} \\
& {\left[Q_{\alpha}^{+}, J^{12}\right]=\frac{1}{2} Q_{\alpha}^{+} \quad \text { for } \alpha=(1),(3),} \\
& {\left[Q_{\alpha}^{+}, J^{12}\right]=\frac{3}{2} Q_{\alpha}^{+} \quad \text { for } \alpha=(2),(4),} \\
& {\left[Q_{\alpha}^{-}, J^{12}\right]=-\frac{3}{2} Q_{\alpha}^{-} \quad \text { for } \alpha=(1),(3),} \\
& {\left[Q_{\alpha}^{-}, J^{12}\right]=-\frac{1}{2} Q_{\alpha}^{-} \quad \text { for } \alpha=(2),(4),} \\
& {\left[Q_{\alpha}^{3}, J^{12}\right]= \pm \frac{1}{2} Q_{\alpha}^{3},}
\end{aligned}
$$

where $Q_{\alpha}^{ \pm}=(1 / 2)\left(Q_{\alpha}^{1} \pm i Q_{\alpha}^{2}\right)$. Eqs.(9), (10), (13) and (14) mean that the generators $Q_{\alpha}^{0}, Q_{\alpha}^{3}, Q_{\alpha}^{+}$(for $\left.\alpha=(1),(3)\right)$ and $Q_{\alpha}^{-}$(for $\left.\alpha=(2),(4)\right)$ raise or lower the helicity of states by $1 / 2$ as the same discussions in the spin-1/2 SUSY algebra. On the other hand, Eqs.(11) and (12) show that the operators $Q_{\alpha}^{+}$(for $\left.\alpha=(2),(4)\right)$ and $Q_{\alpha}^{-}$(for $\alpha=(1),(3))$ raise or lower the helicity of states by $3 / 2$ in contrast with the case of the spin-1/2 SUSY.

Next from Eq.(1) we study both massless and massive representations of the spin-3/2 SUSY algebra. Let us first discuss on the massless case, $P^{2}=0$. By choosing a light-like reference frame, where $P_{a}=(\epsilon, 0,0, \epsilon)$, Eq.(1) for $Q_{\alpha}^{0}, Q_{\alpha}^{ \pm}, Q_{\alpha}^{3}$ becomes

$$
\begin{aligned}
& \left\{Q_{(1)}^{0}, Q_{(3)}^{+}\right\}=\epsilon, \quad\left\{Q_{(2)}^{0}, Q_{(4)}^{-}\right\}=\epsilon, \\
& \left\{Q_{(3)}^{0}, Q_{(1)}^{+}\right\}=-\epsilon, \quad\left\{Q_{(4)}^{0}, Q_{(2)}^{-}\right\}=-\epsilon, \\
& \left\{Q_{(1)}^{3}, Q_{(3)}^{+}\right\}=-\epsilon, \quad\left\{Q_{(2)}^{3}, Q_{(4)}^{-}\right\}=-\epsilon, \\
& \left\{Q_{(3)}^{3}, Q_{(1)}^{+}\right\}=\epsilon, \quad\left\{Q_{(4)}^{3}, Q_{(2)}^{-}\right\}=\epsilon, \\
& \left\{Q_{(2)}^{+}, Q_{(3)}^{-}\right\}=\epsilon, \quad\left\{Q_{(2)}^{-}, Q_{(3)}^{+}\right\}=-\epsilon,
\end{aligned}
$$

and all other anticommutators vanish. Note that the 14 generators appear in Eq.(15) (i.e., the two generators, $Q_{(4)}^{+}$and $Q_{(1)}^{-}$, do not appear). In order to find an example of the generators in the Fock space, we divide Eq.(15) into the following three 
(irreducible) parts,

$$
\begin{aligned}
& \left\{Q_{(1)}^{0}, Q_{(3)}^{+}\right\}=\epsilon, \quad\left\{Q_{(4)}^{0}, Q_{(2)}^{-}\right\}=-\epsilon, \\
& \left\{Q_{(1)}^{3}, Q_{(3)}^{+}\right\}=-\epsilon, \quad\left\{Q_{(4)}^{3}, Q_{(2)}^{-}\right\}=\epsilon, \\
& \left\{Q_{(2)}^{-}, Q_{(3)}^{+}\right\}=-\epsilon,
\end{aligned}
$$

and

$$
\begin{aligned}
& \left\{Q_{(2)}^{0}, Q_{(4)}^{-}\right\}=\epsilon, \quad\left\{Q_{(3)}^{0}, Q_{(1)}^{+}\right\}=-\epsilon, \\
& \left\{Q_{(2)}^{3}, Q_{(4)}^{-}\right\}=-\epsilon, \quad\left\{Q_{(3)}^{3}, Q_{(1)}^{+}\right\}=\epsilon
\end{aligned}
$$

for the generators which raise or lower the helicity of states by $1 / 2$, while

$$
\left\{Q_{(2)}^{+}, Q_{(3)}^{-}\right\}=\epsilon
$$

for the generators which raise or lower the helicity of states by $3 / 2$. We further define creation and annihilation operators from appropriately rescaled generators as

$$
\begin{aligned}
& a_{1}=\frac{1}{\sqrt{\epsilon}}\left(\xi_{1} Q_{(1)}^{0}+\xi_{2} Q_{(1)}^{3}+\xi_{3} Q_{(2)}^{-}\right), \\
& a_{1}^{\dagger}=\frac{1}{\sqrt{\epsilon}}\left(-\xi_{1} Q_{(4)}^{0}-\xi_{2} Q_{(4)}^{3}+\xi_{3} Q_{(3)}^{+}\right), \\
& a_{2}=\frac{1}{\sqrt{\epsilon}}\left(\xi_{1}^{\prime} Q_{(3)}^{0}+\xi_{2}^{\prime} Q_{(3)}^{3}+\xi_{3}^{\prime} Q_{(4)}^{-}\right), \\
& a_{2}^{\dagger}=\frac{1}{\sqrt{\epsilon}}\left(\xi_{1}^{\prime} Q_{(2)}^{0}+\xi_{2}^{\prime} Q_{(2)}^{3}-\xi_{3}^{\prime} Q_{(1)}^{+}\right), \\
& a_{3}=\frac{1}{\sqrt{\epsilon}} Q_{(3)}^{-}, \\
& a_{3}^{\dagger}=\frac{1}{\sqrt{\epsilon}} Q_{(2)}^{+},
\end{aligned}
$$

which are consistent with the Majorana condition of $Q_{\alpha}^{a}$. $\S$ In Eq.(19) $\left(\xi_{1}, \xi_{2}, \xi_{3}\right)$ and $\left(\xi_{1}^{\prime}, \xi_{2}^{\prime}, \xi_{3}^{\prime}\right)$ are arbitrary parameters which can be chosen as $\left\{a_{i}, a_{i}^{\dagger}\right\}=1$ for $i=1,2$.

§In the two-component spinor formalism, the components, $\left(Q_{(1)}^{a}, Q_{(2)}^{a}\right)$, correspond to an undotted spinor, while the $\left(Q_{(3)}^{a}, Q_{(4)}^{a}\right)$ are expressed as a dotted spinor. Also the Majorana condition of $Q_{\alpha}^{a}$ means $\left(Q_{(1)}^{a}\right)^{\dagger}=-Q_{(4)}^{a}$ and $\left(Q_{(2)}^{a}\right)^{\dagger}=Q_{(3)}^{a}$ by the hermitian (complex) conjugation (for example, see [10]). 
Then Eqs.(16) to (18) become the following anticommutation relations,

$$
\begin{aligned}
& \left\{a_{1}, a_{1}^{\dagger}\right\}=\left\{2\left(\xi_{1}-\xi_{2}\right)-\xi_{3}\right\} \xi_{3}, \\
& \left\{a_{2}, a_{2}^{\dagger}\right\}=2\left(\xi_{1}^{\prime}-\xi_{2}^{\prime}\right) \xi_{3}^{\prime}, \\
& \left\{a_{i}, a_{j}\right\}=0, \quad\left\{a_{i}^{\dagger}, a_{j}^{\dagger}\right\}=0,
\end{aligned}
$$

where $i, j=1,2$, while

$$
\begin{aligned}
& \left\{a_{3}, a_{3}^{\dagger}\right\}=1, \\
& \left\{a_{3}, a_{3}\right\}=0, \quad\left\{a_{3}^{\dagger}, a_{3}^{\dagger}\right\}=0 .
\end{aligned}
$$

Namely, Eqs.(20) and (21) are equivalent to Eq.(15) under the definition (19), although the physical meaning and the mathematical structure of Eq.(19) are not known.

If we choose the values of $\left(\xi_{1}, \xi_{2}, \xi_{3}\right)$ and $\left(\xi_{1}^{\prime}, \xi_{2}^{\prime}, \xi_{3}^{\prime}\right)$ in Eq. (20) as $\left\{a_{i}, a_{i}^{\dagger}\right\}=1$ for $i=1,2$, the $\left(a_{i}, a_{i}^{\dagger}\right)$ mean the operators in the Fock space which raise or lower the helicity of states by $1 / 2$. Also the $\left(a_{3}, a_{3}^{\dagger}\right)$ in Eq. $(21)$ are the operators in the Fock space which raise or lower the helicity of states by $3 / 2$. Therefore, a massless irreducible representation for the spin-3/2 SUSY algebra induced from Eqs.(20) and (21) is

$$
\left[\underline{1}\left(+\frac{3}{2}\right), \underline{2}(+1), \underline{1}\left(+\frac{1}{2}\right), \underline{1}(0), \underline{2}\left(-\frac{1}{2}\right), \underline{1}(-1)\right]+[\text { CPT conjugate }]
$$

In Eq. $(22) \underline{n}(\lambda)$ means the number of states $n$ for the helicity $\lambda$.

Let us second investigate the algebra (1) for the massive case, $P^{2}=m^{2}$. By taking a rest frame momentum to be $P_{a}=(m, 0,0,0)$, Eq.(1) for $Q_{\alpha}^{ \pm}, Q_{\alpha}^{3}$ becomes

$$
\begin{aligned}
& \left\{Q_{(1)}^{3}, Q_{(3)}^{+}\right\}=-m, \quad\left\{Q_{(2)}^{3}, Q_{(4)}^{-}\right\}=-m, \\
& \left\{Q_{(3)}^{3}, Q_{(1)}^{+}\right\}=m, \quad\left\{Q_{(4)}^{3}, Q_{(2)}^{-}\right\}=m, \\
& \left\{Q_{(1)}^{+}, Q_{(4)}^{-}\right\}=-\frac{1}{2} m, \quad\left\{Q_{(1)}^{-}, Q_{(4)}^{+}\right\}=\frac{1}{2} m, \\
& \left\{Q_{(2)}^{+}, Q_{(3)}^{-}\right\}=\frac{1}{2} m, \quad\left\{Q_{(2)}^{-}, Q_{(3)}^{+}\right\}=-\frac{1}{2} m,
\end{aligned}
$$

and all other anticommutators vanish. Note that in $\operatorname{Eq}(23) Q_{\alpha}^{0}$ is completely decoupled from the algebra, while the anticommutator $\left\{Q_{(1)}^{+}, Q_{(4)}^{-}\right\}$does not vanish and the two generators, $Q_{(4)}^{+}$and $Q_{(1)}^{-}$, appear in contrast with Eq.(15). 
As in the massless case, we divide Eq.(23) into four (irreducible) parts as

$$
\begin{aligned}
& \left\{Q_{(1)}^{3}, Q_{(3)}^{+}\right\}=-m, \quad\left\{Q_{(4)}^{3}, Q_{(2)}^{-}\right\}=m, \\
& \left\{Q_{(2)}^{-}, Q_{(3)}^{+}\right\}=-\frac{1}{2} m
\end{aligned}
$$

and

$$
\begin{aligned}
& \left\{Q_{(2)}^{3}, Q_{(4)}^{-}\right\}=-m, \quad\left\{Q_{(3)}^{3}, Q_{(1)}^{+}\right\}=m, \\
& \left\{Q_{(1)}^{+}, Q_{(4)}^{-}\right\}=-\frac{1}{2} m
\end{aligned}
$$

for the generators which raise or lower the helicity of states by $1 / 2$, while

$$
\left\{Q_{(2)}^{+}, Q_{(3)}^{-}\right\}=\frac{1}{2} m
$$

and

$$
\left\{Q_{(1)}^{-}, Q_{(4)}^{+}\right\}=\frac{1}{2} m
$$

for the generators which raise or lower the helicity of states by $3 / 2$. We also define creation and annihilation operators from appropriately rescaled generators as

$$
\begin{aligned}
& a_{1}=\frac{1}{\sqrt{m}}\left(\eta_{1} Q_{(1)}^{3}+\eta_{2} Q_{(2)}^{-}\right) \\
& a_{1}^{\dagger}=\frac{1}{\sqrt{m}}\left(-\eta_{1} Q_{(4)}^{3}+\eta_{2} Q_{(3)}^{+}\right), \\
& a_{2}=\frac{1}{\sqrt{m}}\left(\eta_{1}^{\prime} Q_{(3)}^{3}+\eta_{2}^{\prime} Q_{(4)}^{-}\right), \\
& a_{2}^{\dagger}=\frac{1}{\sqrt{m}}\left(\eta_{1}^{\prime} Q_{(2)}^{3}-\eta_{2}^{\prime} Q_{(1)}^{+}\right), \\
& a_{3}=\sqrt{\frac{2}{m}} Q_{(3)}^{-}, \\
& a_{3}^{\dagger}=\sqrt{\frac{2}{m}} Q_{(2)}^{+}, \\
& a_{4}=-\sqrt{\frac{2}{m}} Q_{(1)}^{-} \\
& a_{4}^{\dagger}=\sqrt{\frac{2}{m}} Q_{(4)}^{+}
\end{aligned}
$$


which are consistent with the Majorana condition of $Q_{\alpha}^{a}$. In Eq. $(28)\left(\eta_{1}, \eta_{2}\right)$ or $\left(\eta_{1}^{\prime}, \eta_{2}^{\prime}\right)$ are arbitrary parameters which can be chosen as $\left\{a_{i}, a_{i}^{\dagger}\right\}=1$ for $i=1,2$.

Then Eqs.(24) to (27) become the following anticommutation relations,

$$
\begin{aligned}
& \left\{a_{1}, a_{1}^{\dagger}\right\}=-\left(2 \eta_{1}+\frac{1}{2} \eta_{2}\right) \eta_{2}, \\
& \left\{a_{2}, a_{2}^{\dagger}\right\}=-\left(2 \eta_{1}^{\prime}-\frac{1}{2} \eta_{2}^{\prime}\right) \eta_{2}^{\prime}, \\
& \left\{a_{i}, a_{j}\right\}=0, \quad\left\{a_{i}^{\dagger}, a_{j}^{\dagger}\right\}=0,
\end{aligned}
$$

where $i, j=1,2$, while

$$
\begin{aligned}
& \left\{a_{3}, a_{3}^{\dagger}\right\}=1 \\
& \left\{a_{4}, a_{4}^{\dagger}\right\}=-1 \\
& \left\{a_{i}, a_{j}\right\}=0, \quad\left\{a_{i}^{\dagger}, a_{j}^{\dagger}\right\}=0
\end{aligned}
$$

where $i, j=3,4$.

For the massive case, we choose the value of $\left(\eta_{1}, \eta_{2}\right)$ in Eq.(29) as $\left\{a_{1}, a_{1}^{\dagger}\right\}=1$, while the value of $\left(\eta_{1}^{\prime}, \eta_{2}^{\prime}\right)$ as $\left\{a_{2}, a_{2}^{\dagger}\right\}$ gives the negative norm, i.e., $\left\{a_{2}, a_{2}^{\dagger}\right\}=-1$. ฯ Then the only $\left(a_{1}, a_{1}^{\dagger}\right)$ mean the operators in the Fock space which raise or lower the helicity of states by $1 / 2$. Also the $\left(a_{3}, a_{3}^{\dagger}\right)$ in Eq. (30) are the operators in the Fock space which raise or lower the helicity of states by $3 / 2$, while the $\left\{a_{4}, a_{4}^{\dagger}\right\}$ in Eq.(30) gives the negative norm. Therefore, a (physical) massive irreducible representation for the spin-3/2 SUSY algebra induced from $\left(a_{1}, a_{1}^{\dagger}\right)$ and $\left(a_{3}, a_{3}^{\dagger}\right)$ uniquely has the following structure,

$$
\left[\underline{1}\left(+\frac{3}{2}\right), \underline{1}(+1), \underline{1}(0), \underline{1}\left(-\frac{1}{2}\right)\right]+[\text { CPT conjugate }]
$$

which represents massive spin-3/2, vector and scalar fields on shell.

Explicit L realizations for the massless or the massive representations, e.g., Eqs.(22) or (31), are now under investigation.

Finally we comment on the systematic linearization of the spin-3/2 NL SUSY in the superspace formalism by introducing a spinor-vector Grassmann coordinate $\theta^{a}$. Indeed, let us denote a $\mathrm{L}$ superfield on the superspace coordinates $\left(x^{a}, \theta^{a}\right)$ by

${ }^{\top}$ Athough the values of $\left(\eta_{1}, \eta_{2}\right)$ and $\left(\eta_{1}^{\prime}, \eta_{2}^{\prime}\right)$ can be chosen as $\left\{a_{i}, a_{i}^{\dagger}\right\}=1$ for $i=1,2$, the excess of helicity- \pm 1 states in the resultant irreducible representation is not adequate for the massive case. 
$\Phi\left(x^{a}, \theta^{a}\right)$, and define specific supertranslations as

$$
\begin{aligned}
& x^{\prime a}=x^{a}-\kappa \epsilon^{a b c d} \bar{\theta}_{b} \gamma_{5} \gamma_{c} \psi_{d}, \\
& \theta^{\prime a}=\theta^{a}-\kappa \psi^{a}
\end{aligned}
$$

which are just the spin-3/2 SUSY version of the specific supertranslations introduced in $[3,5]$. Then we can prove that the superfield on $\left(x^{\prime a}, \theta^{\prime a}\right)$, i.e., $\Phi\left(x^{\prime a}, \theta^{\prime a}\right)=$ $\tilde{\Phi}\left(x^{a}, \theta^{a} ; \psi^{a}(x)\right)$, transforms homogeneously; namely, according to superspace translations of $\left(x^{a}, \theta^{a}\right)$ generated by the global (Majorana) spinor-vector parameter $\zeta^{a}$, $\|$

$$
\begin{aligned}
x^{\prime a} & =x^{a}+\epsilon^{a b c d} \bar{\theta}_{b} \gamma_{5} \gamma_{c} \zeta_{d}, \\
\theta^{\prime a} & =\theta^{a}+\zeta^{a} .
\end{aligned}
$$

in addition to the spin-3/2 NL SUSY transformation (5), the $\tilde{\Phi}$ transforms as

$$
\delta_{\zeta} \tilde{\Phi}=\xi^{a} \partial_{a} \tilde{\Phi}
$$

where $\xi^{a}=\kappa \epsilon^{a b c d} \bar{\zeta}_{b} \gamma_{5} \gamma_{c} \psi_{d}$. Eq. (34) means that the components of $\tilde{\Phi}$ do not transform into each other as in the case of the spin-1/2 SUSY $[3,5]$, and so the following conditions,

$$
\text { components of } \tilde{\Phi}=\text { constant }
$$

may give SUSY invariant relations which connect a spin-3/2 L SUSY action, if it exists, with the spin-3/2 NL SUSY one (8).

We summarize our results as follows. We have studied the unitary representation of the spin-3/2 SUSY algebra introduced in [7] for both massless and massive cases. The role of the spinor-vector generator $Q_{\alpha}^{a}$ as creation and annihilaiton operators, which raise or lower the helicity of states by $1 / 2$ or by $3 / 2$, has been disscussed explicitly in Eqs. from (9) to (14). By defining creation and annihilation operators from appropriately rescaled generators as in Eqs.(19) or (28), the structure of the L supermultiplets induced from the spin-3/2 SUSY algebra has been shown as Eq.(22) for the massless case or Eq.(31) for the massive case. We have also shown the systematic linearization method by introducing the specific supertranslations of Eq.(32).

${ }^{\|}$The specific supertranslations (32) correspond to the supertranslations (33) by replacing the $\zeta^{a}$ with $-\kappa \psi^{a}$, while the NL SUSY transformation (5) (or Eq.(4)) is defined on a hypersuface $\theta^{a}=\kappa \psi^{a}$ in Eq.(33). 


\section{References}

[1] J. Wess and B. Zumino, Phys. Lett. B49 (1974) 52.

[2] D.V. Volkov and V.P. Akulov, JETP Lett. 16 (1972) 438; Phys. Lett. B46 (1973) 109.

[3] E.A. Ivanov and A.A. Kapustnikov, Relation between linear and nonlinear realizations of supersymmetry, JINR Dubna Report No. E2-10765, 1977 (unpublished);

E.A. Ivanov and A.A. Kapustnikov, J. Phys. A11 (1978) 2375; J. Phys. G8 (1982) 167.

[4] M. Roček, Phys. Rev. Lett. 41 (1978) 451.

[5] T. Uematsu and C.K. Zachos, Nucl. Phys. B201 (1982) 250.

[6] K. Shima, Y. Tanii and M. Tsuda, Phys. Lett. B525 (2002) 183; Phys. Lett. B546 (2002) 162.

[7] N.S. Baaklini, Phys. Lett. B67 (1967) 335.

[8] K. Shima, Z. Phys. C18 (1983) 25; European Phys. J. C7 (1999) 341; Phys. Lett. B501 (2001) 237.

[9] S. Deser and B. Zumino, Phys. Rev. Lett. 38 (1977) 1433.

[10] J. Wess and J. Baggar, Supersymmetry and Supergravity, Princeton Univ. Press, Princeton, NJ, 1992. 